\title{
Research Paper \\ The Study of Some Religious Correlates of Death Depression Among University Students
}

\section{Ali Mohammadzadeh ${ }^{1}$, Ahmad Ashouri ${ }^{2 *}$}

1. PhD in Psychology, Associate Professor, Department of Psychology, Payam-e Noor University, Tehran, Iran

2. PhD in Clinical Psychology, Assistant Professor, Department of Clinical Psychology, School of Behavioral Sciences \& Mental Health (Tehran Institute of Psychiatry), Iran University of Medical Sciences, Tehran, Iran.

Received: 22 Sep. 2016 Accepted: 21 Feb. 2017
Key words:

Death depression, Attachment style, Religious beliefs, Religious coping

\section{A B STRACT}

Objectives Death depression consists of death despair, death loneliness, death sadness, death dread, and death finality. The present study was carried out with the aim of investigating some religious correlates of death depression.

Methods In this descriptive analytical study, a sample of 347 participants was selected using stratified sampling method. The participants responded to death obsession, acting on religious beliefs, religious coping, and attachment to God Scales. Data were analyzed using multiple regression analysis.

Results Among the religious variables, insecure attachment to God and negative religious coping showed high contribution in predicting death depression.

Conclusion When the participants faced real-life events, those who used negative coping strategies experienced higher death depression. In addition, those who considered God Asa source of punishment rather thana source of love and remained doubtful and anxious in accepting God as a secure base showed higher death depression.

\section{Extended Abstract}

\section{Introduction}

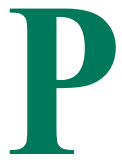

redicting death of the self and fear of death process of the important people in life creates morbid reactions in some people. In studies regarding death, these reactions are classified in three dimensions, i.e.,death anxiety, death obsession, and death depression. Studies have showed that religion and religiosity are causes of reduction in some conditions, stimulates death phobic attitudes [19]. In connection with the innovative aspect of this study, a review of the literature suggests that few studies have been done in the area of death depression and psychological-religious fac- tors related to it, especially in Iran. Due to the lack of findings and also with regard to the religious variables in explaining the fact that death depression can have a decisive role,the goal of this research is to investigate some religious study correlates of death depression, including the practice of religious beliefs, religious harmony, and attachment to God.

\section{Method}

The study was a descriptive-analytical study. The study population consisted of all PNU students of East Azerbaijan Province enrolled in the academic year of 2014-15. Stratified random sampling was used to select the subjects. Given the nature of research and considering the adequacy of subject numbers in the research, the sample size was set at 347 . Af-

\section{* Corresponding Author:}

Ahmad Ashouri, PhD

Address: Department of Clinical Psychology, School of Behavioral Sciences (Tehran Institute of Psychiatry), Iran University of Medical Sciences, Tehran, Iran.

Tel: +98 (21) 66506853

E-mail: ahmad.ashouri@gmail.com 
ter describing the research objectives, consent was obtained from the participants to participate in the study. Research questionnaires were given to them. An arrangement was made for simultaneous completion of questionnaires, where half of the participants first completed some scales and the other half completed the other scales. In this way, the effects of the completion order were controlled.

For data analysis, simultaneous multiple regression analysis was performed using SPSS (v. 21) software. Research tools included Death depression scale,Questionnaire to practice religious beliefs of the temple, Religious coping short form scale, and scale of attachment to God.

\section{Results}

Demographic information of sample is shown in Table 1. According to the table, the number of female participants (235) is comparable to the number of male participants (104) in this study. Eleven subjects did not specify their gender. Also, the mean scores of the variables of the study and correlations between death obsession and religious components are summarized in Table 2.

According to the table, there is no significant relationship between death depression and different dimensions of practicing religious beliefs. It was observed that death depression has a direct relationship with negative religious coping styles and anxious attachment to God and an inverse relationship with religious coping styles. The multiple linearity assumption for the assessment of independence was investigated for predictive variables. The software gave tolerance index as 0.87 to 1 and variance inflation factor index as 1 to 1.14 . It can be accepted that predictive variables are independent of each other, and there was no multiple linearity. Data normalization was evaluated using the Kolmogorov-Smirnov test, and the results showed that there was no deviation from the normal value $($ Coefficient $=0.035, \mathrm{P}<0.2$ ).

Regression analysis results are provided among the variables in Table 3. Based on these results, the F observed was significant $(\mathrm{P}<0.001)$, and $13 \%$ of the variance of the death depression was determined by anxious attachment to God and positive and negative religious coping styles $\left(\mathrm{r}^{2}=0.13\right)$. Regression analysis of predictive variables showed that anxious attachment to God and positive and negative religious coping styles can significantly explain depression death variance. Impact coefficient of anxious attachment to God was 0.27 and that of positive and negative religious coping styles were 0.33 and -0.39 , respectively. The T-Statistics suggested that these variables can predict changes related to death depression with more than $95 \%$ confidence. This means that increasing anxiety attachment to God and negative religious coping style led to increased death depression. In addition,

Table 1. Demographic sample

\begin{tabular}{cccc}
\hline & Frequency (\%) & Mean of the Age & Standard Deviation \\
\hline Female group & $235(67.1)$ & 26.75 & 7.55 \\
Male group & $104(29.7)$ & 28.54 & 8.99 \\
Total & $339(96.8)$ & 27.30 & 8.05 \\
\hline
\end{tabular}

Iranian Journal of
PSYCHIATRY AND CUINICAL PSYCHOLOGY

Table 2. The mean scores and the correlation between the variables of the study

\begin{tabular}{ccc}
\hline Variable & Mean (SD) & Coefficient of Correlation With Death Obsession \\
\hline Death depression & $31.79(14.54)$ & 1 \\
\hline Practicing religious beliefs & $56.39(20.43)$ & -0.36 \\
\hline Practice religious beliefs (motivation and relationships) & $44.54(16.10)$ & 0.09 \\
\hline Practice religious beliefs (performing obligatory acts) & $7.65(2.94)$ & 0.03 \\
Practice religious beliefs (performing non-obligatory acts) & $4.34(2.81)$ & 0.10 \\
Positive religious coping & $14.47(4.50)$ & $-0.10(\mathrm{P}<0.025)$ \\
Negative religious coping & $5.79(4.64)$ & $0.30(\mathrm{P}<0.001)$ \\
Anxious attachment to God & $40.26(9.78)$ & $0.32(\mathrm{P}<0.001)$ \\
Avoidant attachment to God & $42.20(9.78)$ & $0.12(\mathrm{P}<0.02)$ \\
\hline
\end{tabular}


Table 3. Regression analysis of death depression on the positive and negative religious coping styles and anxious attachment to God

\begin{tabular}{|c|c|c|c|c|c|c|c|c|c|}
\hline Criterion Variable & Predictive Variables & $\mathbf{r}$ & $\mathbf{R}^{2}$ & $\mathbf{F}$ & Sig. & B & $\boldsymbol{\beta}$ & $\mathbf{t}$ & Sig. \\
\hline \multirow{3}{*}{ Death depression } & Anxious attachment to God & & & & & 0.27 & 0.28 & 5.25 & 0.001 \\
\hline & Negative religious coping styles & 0.35 & 0.13 & 16.46 & 0.001 & 0.33 & 0.10 & 1.94 & 0.05 \\
\hline & Positive religious coping styles & & & & & -0.39 & -0.12 & -2.39 & 0.02 \\
\hline
\end{tabular}

decreased positive coping style also led to increased death depression.

\section{Discussion}

The results of the religious variables studied showed that anxious attachment to God (comprised of an avoidant and anxious attachment style) and negative religious coping (directly) and positive religious coping (inversely) have the most explanatory power of death depression. Till date, two studies have been reported which examined the correlates of religious death depression, with which the results of this study are in parallel. The two studies reported an inverse relationship between death depression and faith in God and life after death $[8,9]$.While explaining these results, we can say that those with insecure attachment style to God reflect anxiety, ambivalence or confusion regarding God's will to respond in times of threat and that in their view,God is not responsive and accessible.

Such people have doubts in the acceptance of God as the basis for safety and abutments at times of hardships. Those who have doubts and are anxious about accepting the God as a secure base may not feel the presence of God in every moment of their lives and are always in anxiety of the absence of God in difficult and important situations of their lives. Such people always suffer from a kind of anxiety, which is a sign of being far from God. Considering the fact that all humans sometimes think about death - not from its religious dimension - but perhaps because of the fear that death leaves in man and since it is possible that death is the end of the road for them,they do not have such a strong anchorage like God when in the midst of life.

Hence, death and thoughts about it create a kind of anxiety in them which cast a shadow over their lives, or because they do not know themselves whether they are worthy of the kindness and love of God or can accept death and embrace it. In case of reviewing their past actions and if they have committed any sin and wrong acts, they do not think of themselves worthy of forgiveness by God. Therefore, death becomes a horrible and fearsome phenomenon for them and even thinking about it would make them extremely anxious.
This study showed that those who use positive coping strategies while facing any negative life events have less death depression than those who use negative coping strategies. According to this, there are those who try to make a stronger relational bond with God during the negative events of life or ask God to help them to overcome the anger and rage resulting from the failure. They rely on their religious beliefs in order for their problems to be reduced and be less despaired and sad in relation to the death, hence, resulting in low death depression. Having such image of the Creator is a kind of relief and relaxation and helps reduce discomforts for their losses. The man who knows his God with all the aesthetic characteristics does not surrender in front of the tribulations of life and leaves behind all the difficulties and hardships through his trust in God.

Conversely, those who feel that God has forgotten them when faced with negative life events or even think that the event happened is a punishment from God, and have doubts about the power of God and think God as inhospitable, show higher levels of death depression. Negative religious coping style lacks a deep internal relationship,and it seems that he/she has anxiety attribute with self. In other words,in this kind of religious coping style, the fear of punishment and rejection by God is considered by the person. These people perceive God more as a source of pain and punishment rather than as a source of love and affection. Positive religious coping is based on a loving relationship with God and a sense of spiritual connection with others and believing in purposefulness of the world [27]. In this regard,the finding is consistent with the findings by Pargament on a relationship between positive religious coping and mental health [28], and its negative correlation with decreased depression [29] and reduced stress [30].

\section{Acknowledgments}

Payam-e Noor University of Tehran has financially supported this paper.

\section{Conflict of Interest}

The authors declared no conflicts of interest. 


\title{
بررسى برخى همربستهاى مذهبى افسردَّى مر مَى در بين دانشجويان
}

على محمدزاده'، "احمد عاشورى'

ا - دكتراي روانشتاسى، دانشيار، كروه روانشناسى، دانشُكاه بيامنور، تهران، ايران.

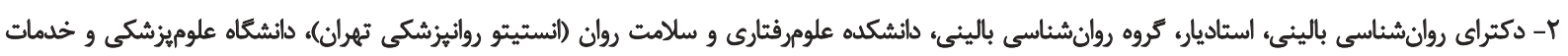

بهاشتى برمانى ايران، تهران، ايران.

\begin{abstract}
حكدن

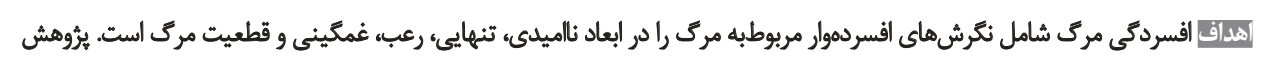

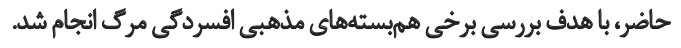

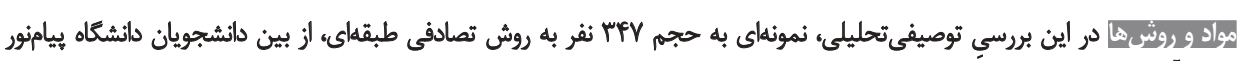

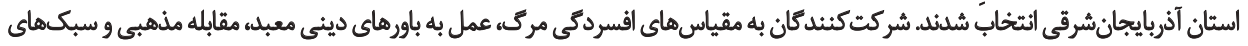

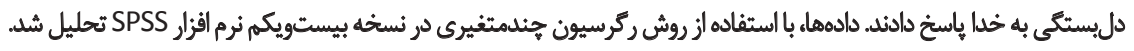

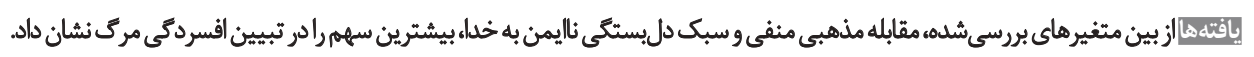

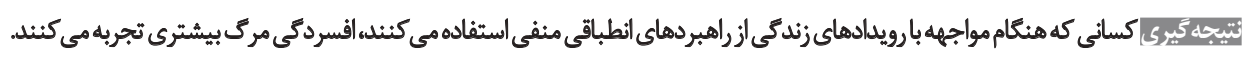

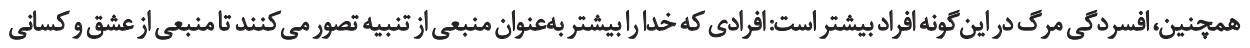

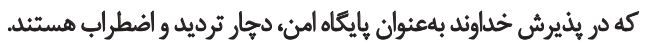

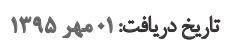

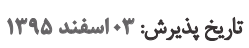

كليدوازٔهها:

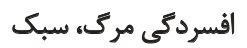

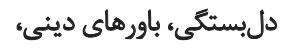
مقابله مذهبي دليدي

افسردگى، مي تواند با ناميدى وجودى و فقدان معنا در زندكى إنى

مقدمه

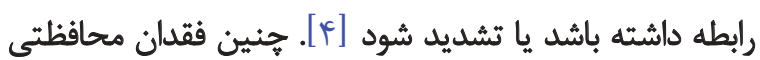

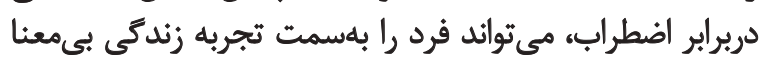

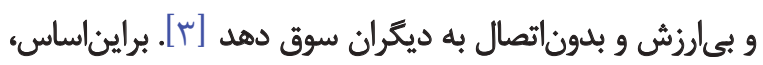

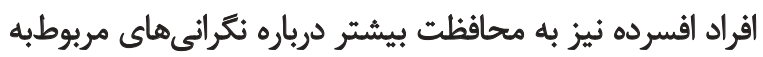

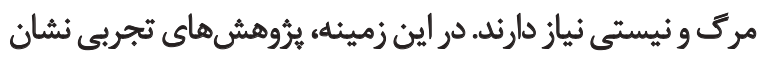

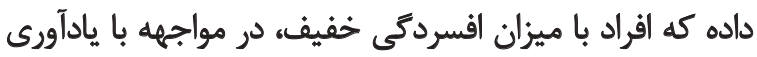

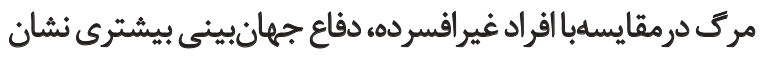

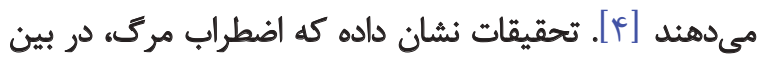

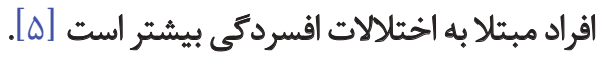

ترس از مرك، ميتواند تهديد درخورتوجهي براي بهزيستى براني

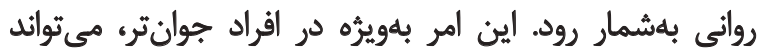

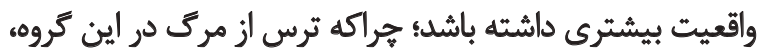

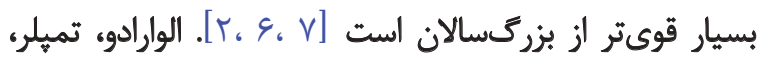

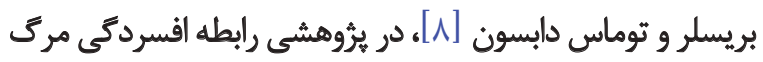

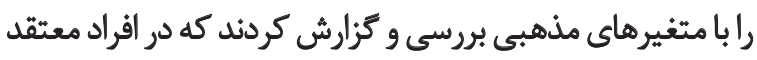

بيشبينى مرى خود و ترس از فرايند مرك و مردن درباره

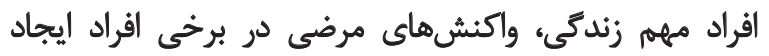

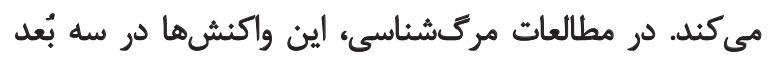

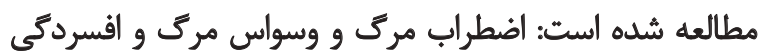

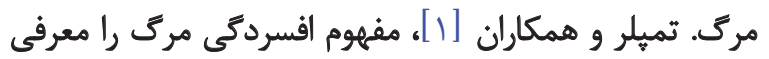

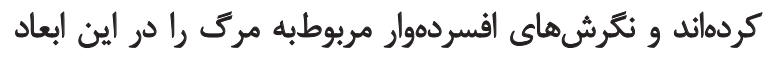

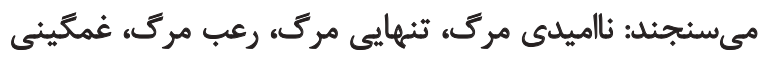

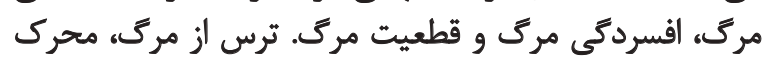

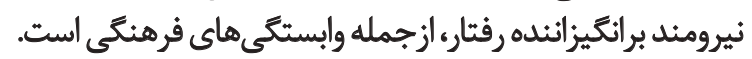

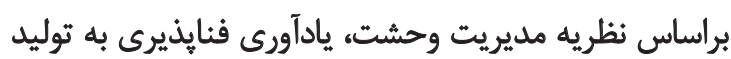

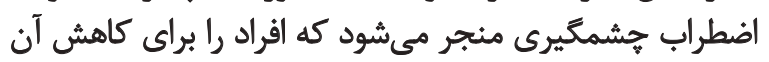

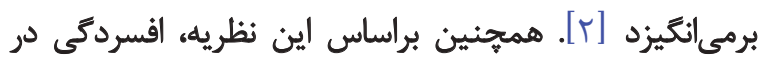

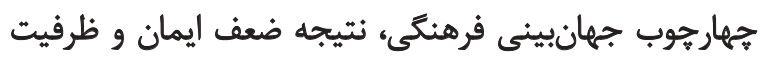

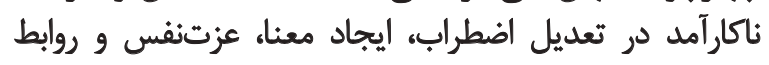

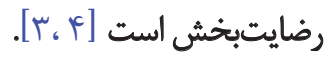

$$
\text { a. }
$$

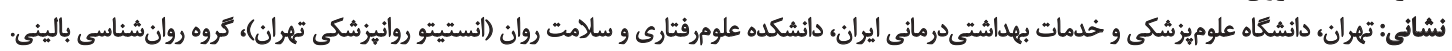
تلفئ:

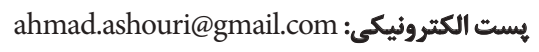




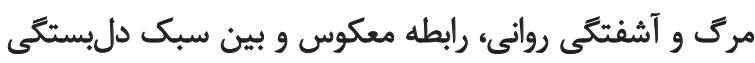

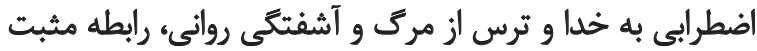
وجود دارد [1/1].

يافتهها، نشان مي دهد كه مذهب و ديندارى در شرايط متفاوت،

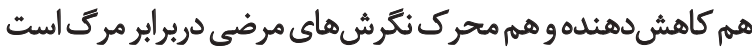

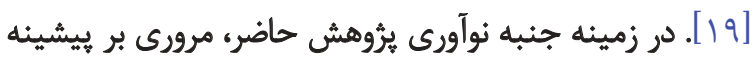

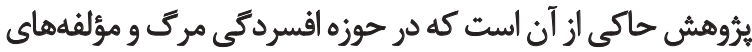

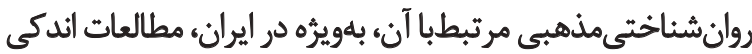

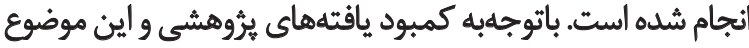

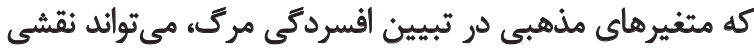

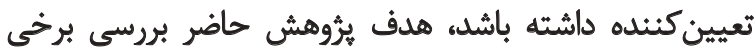

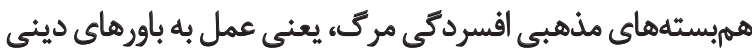

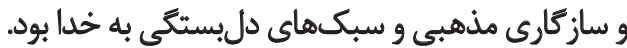

يروهش حاضر از نوع توصيفىتحليلى بود و جامعه آمارى

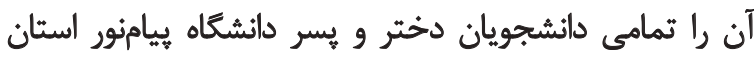

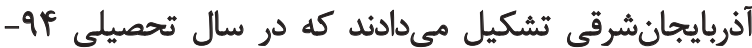

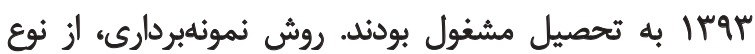

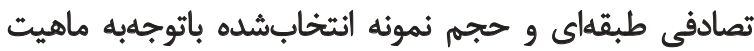

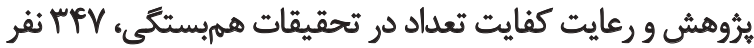

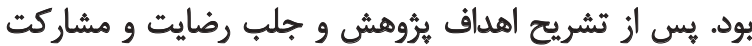

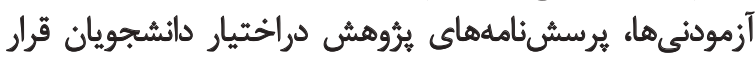

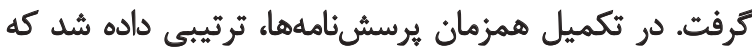

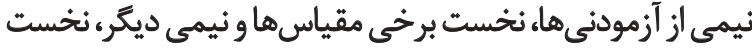

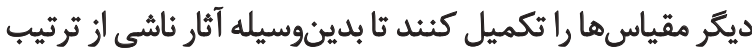

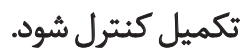

مهمترين ملاك ورود به يروهش، قراركرفتن در سنين

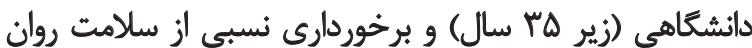

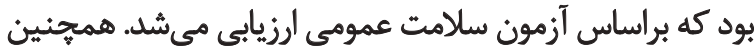

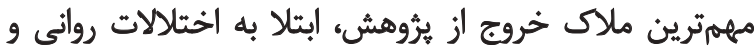

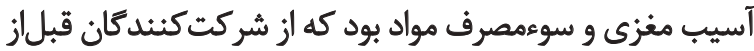

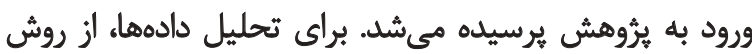

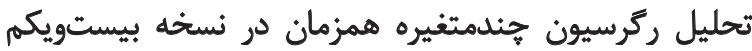

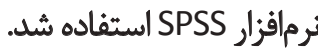

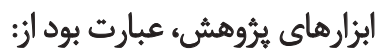

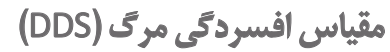

تميلر و همكاران [ [1]، اين مقياس را طراحى كردماند. اين

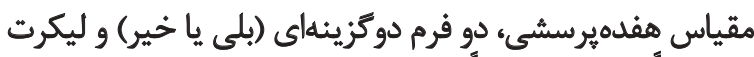

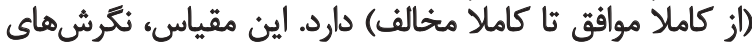

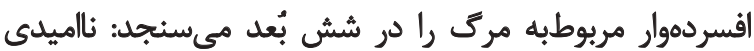

به خدا و زندگى يسازمركى ميزان افسردگى مرى كم است.

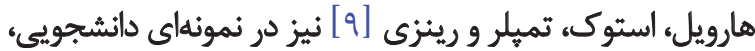

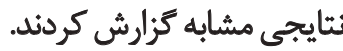

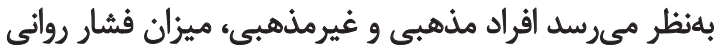

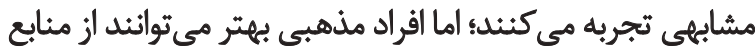

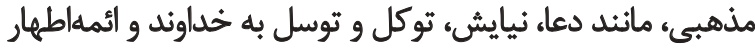

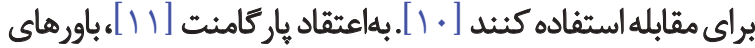

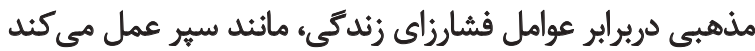

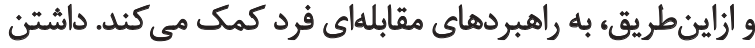

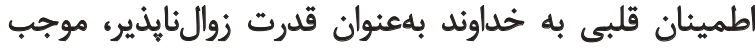

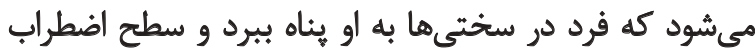

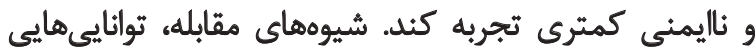

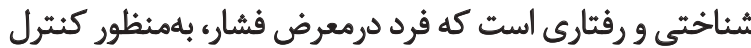

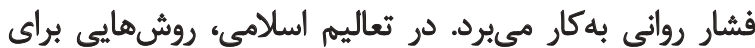

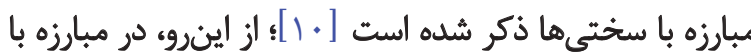

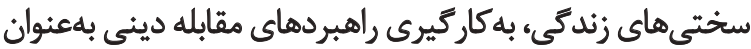

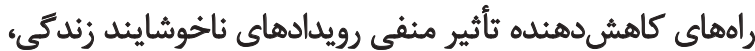

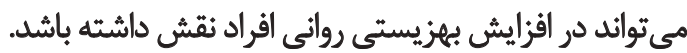
مطالعات نشان ميدهد كه اتصال به منبع غنى قدرت و وييوند

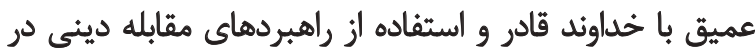

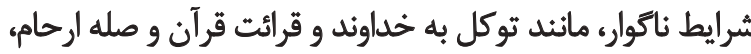

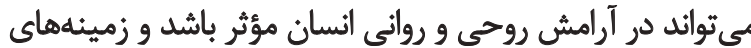

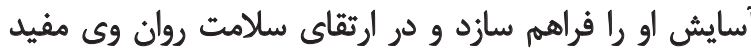

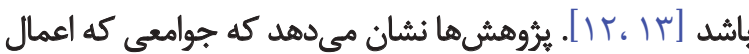

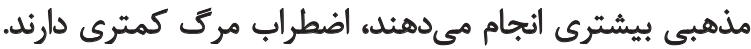

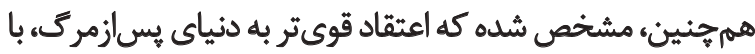

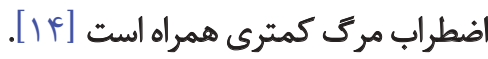

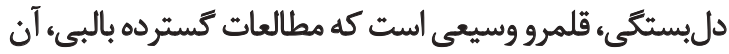

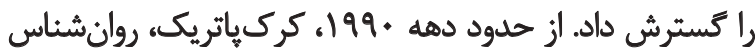

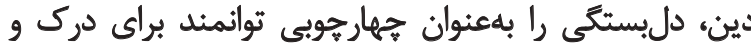

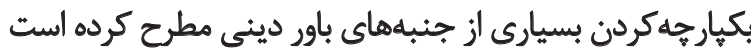

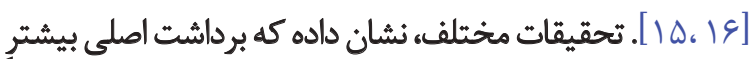

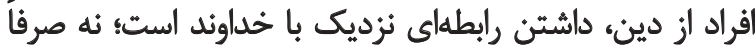

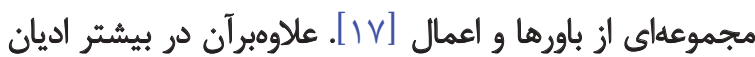

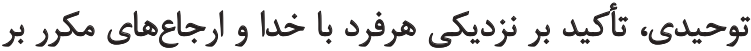

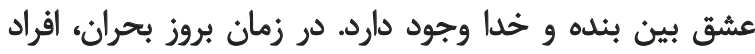

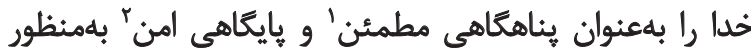

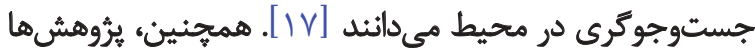

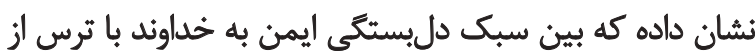

1. Haven of safety

2. Secure base 
زندكى را اندازه مى كيرد. اين مقياس، از دو زيرمقياس مقابله

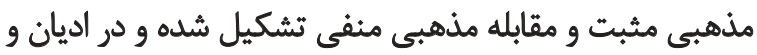

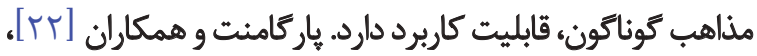

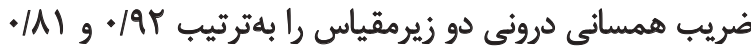

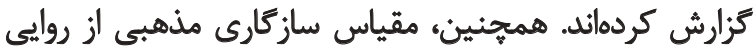

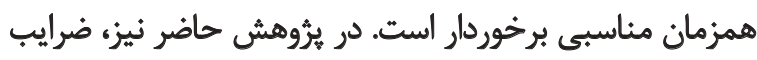

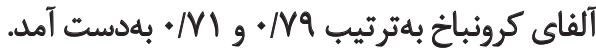

$$
\text { مقياس سبكهاي دلبستئى به خدا }
$$

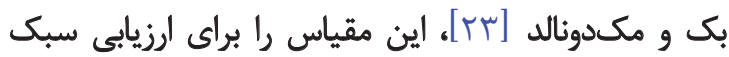

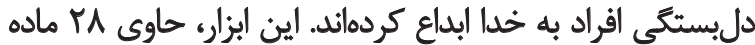

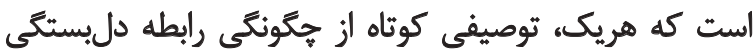

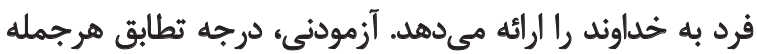

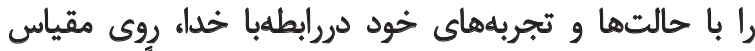

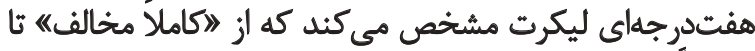

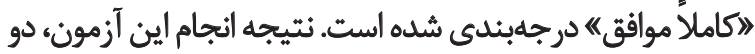

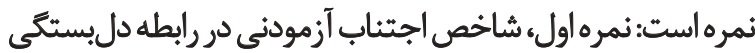
با خدا و نمره دوم، شاخص اضطراب إنداب وي در اين رابطه است.

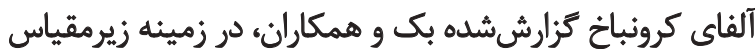

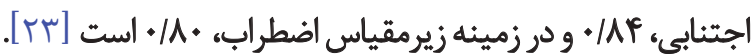

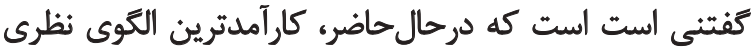

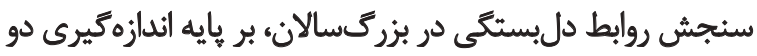

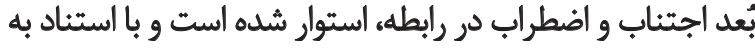

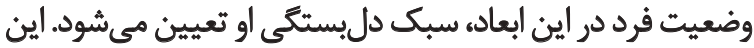

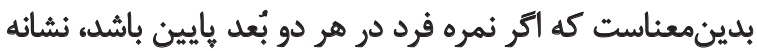

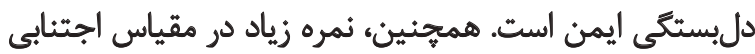

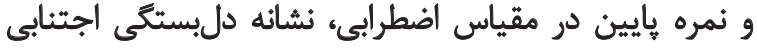

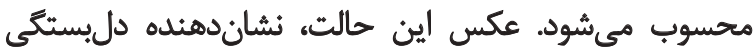

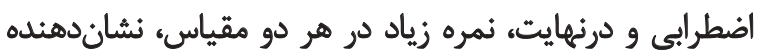

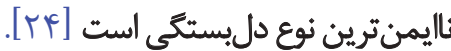

يافتّهها

در جدول شماره ا، ويرٔكىهاى جمعيتشناختى نمونههاى

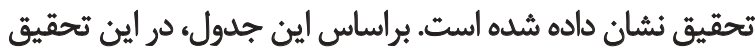

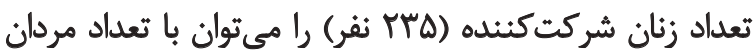

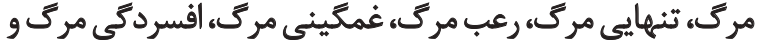

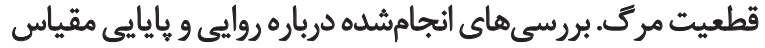

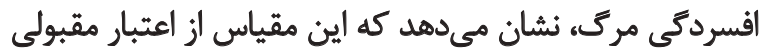

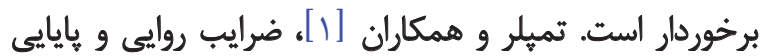

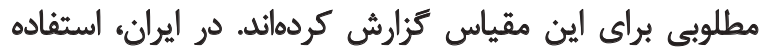

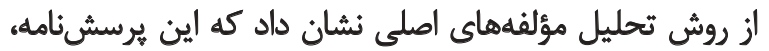

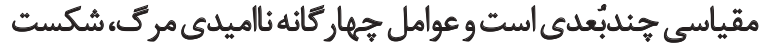

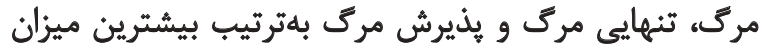

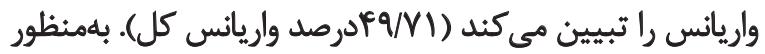

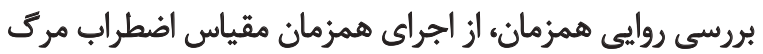

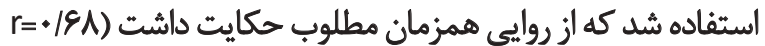

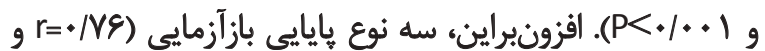

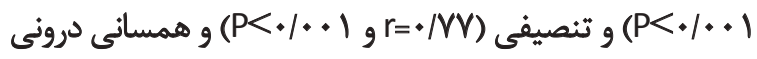

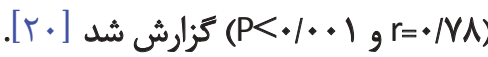

$$
\text { بوسش ناهه عمل به باورهاي دينى معبد }
$$

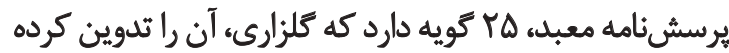

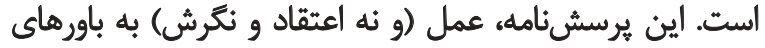

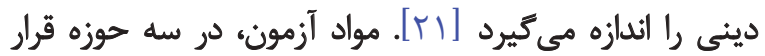

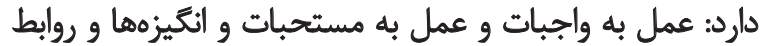

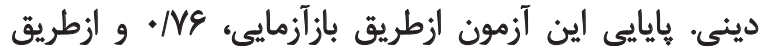

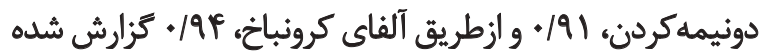

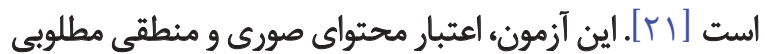

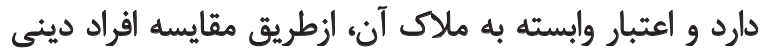

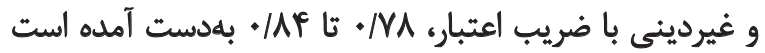

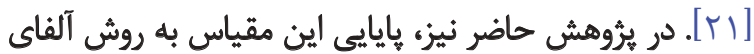

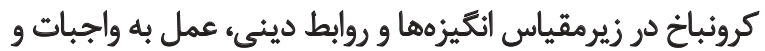

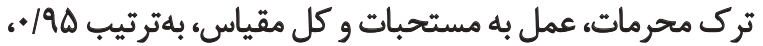

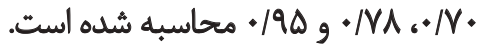

\section{مقياس مقابله مذهبي و فرم كوتاه (B-RCOPE)} اين مقياس جهارده هيرسش دارد و ياركامنت و فيول و بردزى

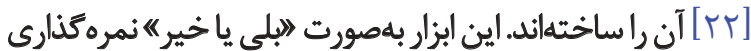

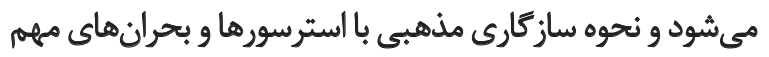

\begin{tabular}{|c|c|c|c|}
\hline انحرافمعيار & ميانكين سنى & فراوانى (درصد) & \\
\hline$V / \Delta \omega$ & re/va & $r^{\prime} \Delta(\Phi / /)$ & كروه مؤنث \\
\hline N99 & KNAP & $1 . P(\mathrm{Tg} / \mathrm{M})$ & كروه مذكر \\
\hline$N+\Delta$ & $\mathrm{W} / \mathrm{r}$. & $\pi$ & كل نمونه \\
\hline
\end{tabular}

جدول ا. ويرّكي هاي جمعيتشناختي نمونه تحقيق

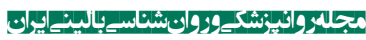


جدول r. ميانكين نمرههاي كسبشده و همربستكى ها در متغيرهاي يروهش

\begin{tabular}{|c|c|c|}
\hline ضريب همبستَّى با وسواس مركى & ميانكين (انحراقمعيار) & مثيخير \\
\hline 1 & $M / N Q(I F / \Delta Y)$ & اقسردكى مرى \\
\hline.$- / 48$ & $\Delta E / T q(T \cdot / R T)$ & عمل به باورهاي ديئى \\
\hline $.1 \cdot 9$ & $\operatorname{Pr} / \Delta \varphi(1 \& / 1)$. & عمل به باورهاي دينى (أكيزهه) و رابطهها) \\
\hline $.1 \cdot r$ & $V / 8 \Delta\left(Y / 9 T^{\natural}\right)$ & عمل به باورهاي دينى (عمل به واجبات) \\
\hline .1 & $P / M T(Y / A))$ & عمل به باورهاى دينى (عمل به مستحبات) \\
\hline$-\cdot / 1 \cdot(\mathrm{P}<\cdot / \cdot r \Delta)$ & $\mid F / F V(\varphi / \Delta \cdot)$ & مقابله مذهبى مثبث \\
\hline$* / \mu \cdot(\mathrm{P}<* / . \cdot 1)$ & $\Delta / V q(f / R T)$ & مقابله مذهبي منفى \\
\hline$\cdot / \pi r(P<\cdot / *+1)$ & $r \cdot / R g(1 \otimes / I Q)$ & دلبستكى اضطرابى به خلا \\
\hline.$/ N(P<\cdot / . r)$ & $F r / r \cdot(q / A)$ & دلبستكى اجتنابي به خدا \\
\hline
\end{tabular}

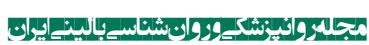

جدول ب. تحليل ركرسيون متغير افسردمى مرك بر سبكهاى مقابله مذهبى مثبت و منفى و دل بستكى اضطرابى به خدا

\begin{tabular}{|c|c|c|c|c|c|c|c|c|c|}
\hline Sig. & $\mathbf{t}$ & b & B & Sig. & $\mathbf{F}$ & $\mathbf{R}^{\mathbf{2}}$ & $\mathbf{r}$ & متغير هاي ييشيين & متغير ملاكى \\
\hline $.1 .+1$ & $\Delta / T \Delta$ &.$/ M A$ & $\cdot / M r$ & & & & & دلبستكى اضطرابى به خلا & \\
\hline+1.0 & $1 / 9 f$ & .11 & - (m & $.1 .+1$ & lepes &.$/ 1 r^{\infty}$ &.$/ r \Delta$ & مقابله مذهبي منفى & اقسردكى مرى \\
\hline $.1 . r$ & $-Y / T q$ &.$- / 1 Y$ &.$- / 17 q$ & & & & & مقابله مأهيى هثبت & \\
\hline
\end{tabular}

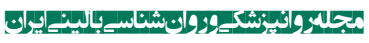

در جدول شماره ب، نتيجه تحليل ركرسيون بين مثنيرهاى

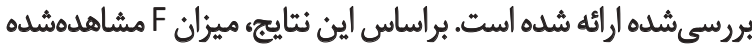

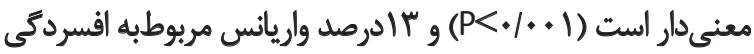

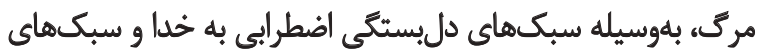

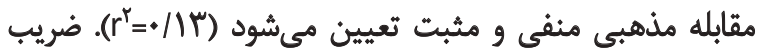

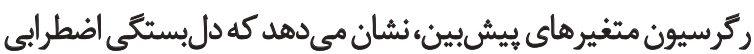

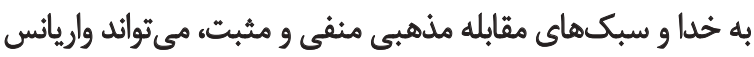
افسردكي مرى را بلهورت معني دار تبيين كند.

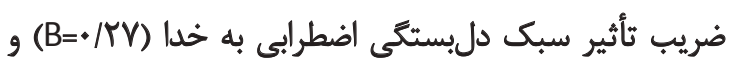

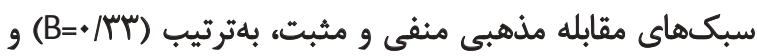

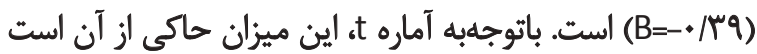

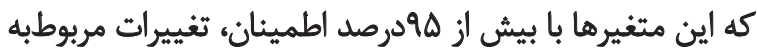

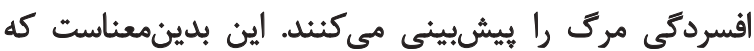

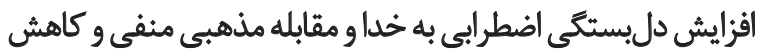

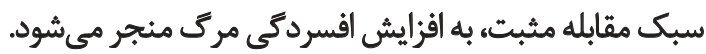

ثب

هدف يروهش حاضر، بررسى ارتباط متغيرهاى مذهبى با

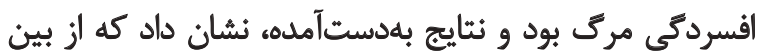

شركت كنئه (F • ( نفر) مقايسه كرد. در اين بررسى، يازده نفر

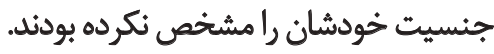

در جدول شماره ؟، ميانگين نمرههاي كسبشده در متغيرهاي

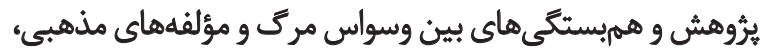

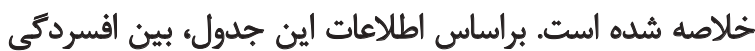

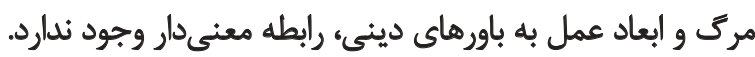

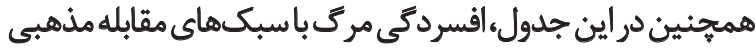

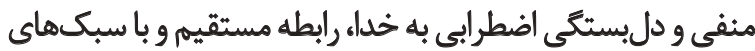

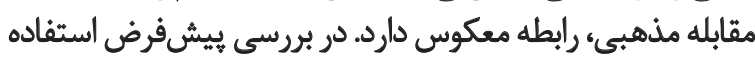

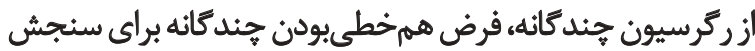

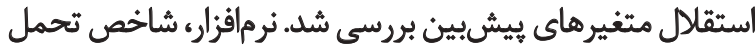

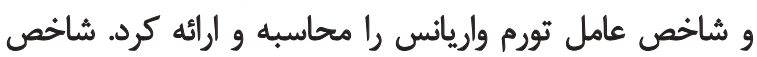

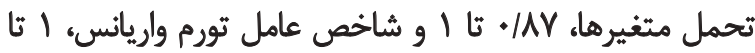

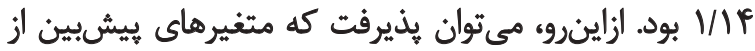

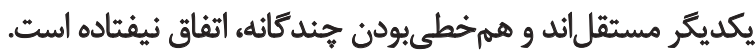

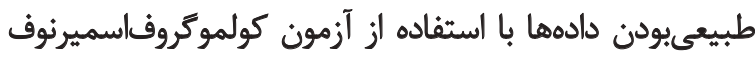

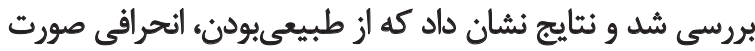

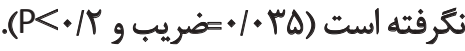


تا موقعيت را بهشكل متفاوتى ارزيابي كنئد. همجنينء توانايي

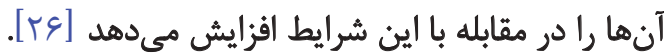

يُروهش حاضر، نشان داد كسانى كه هنكام مواجها باديه

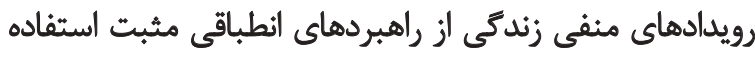

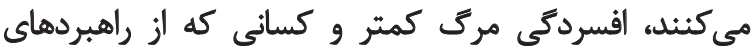

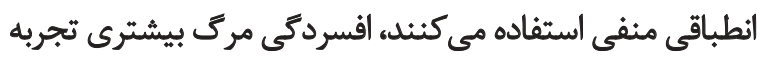

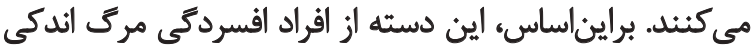

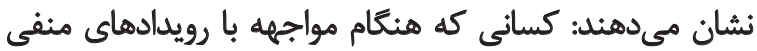

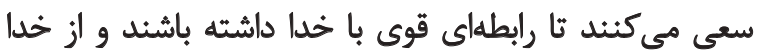

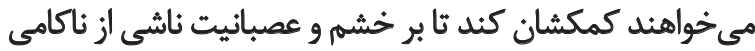

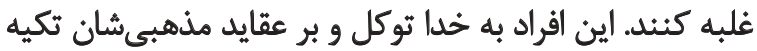

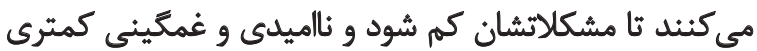

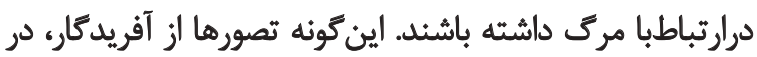

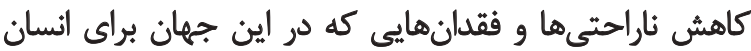

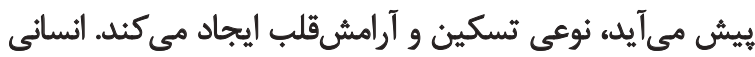

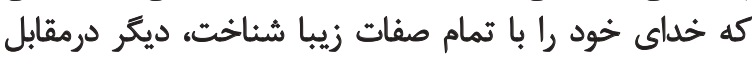

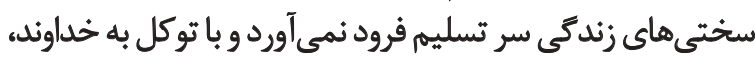
تمامى مشكلات را يشت زئسر مي كذارد.

درمقابل، كسانى كه هنكام مواجهه با رويدادهاي منفي

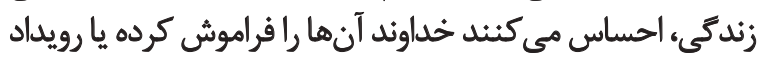

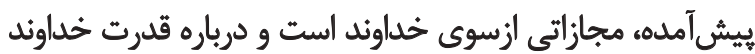

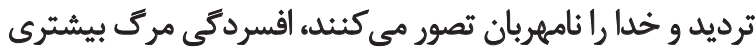

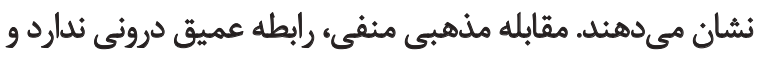

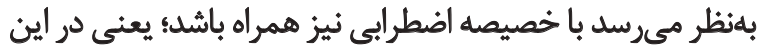

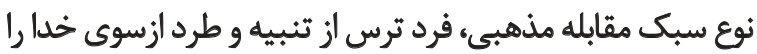

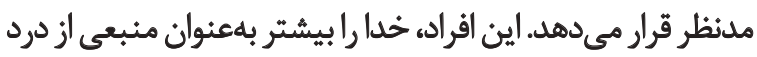

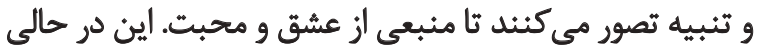

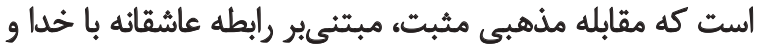

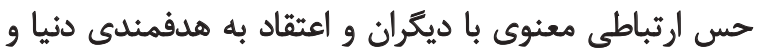

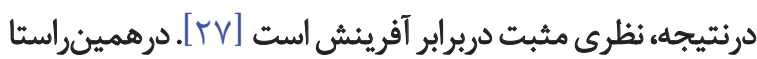

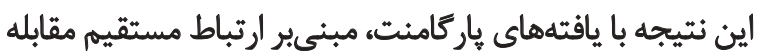

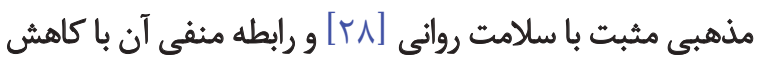

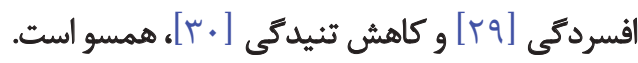

يثروهش حاضر از دونظر محدوديت داشت: ا.نمونه بررسى تشده

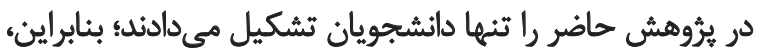

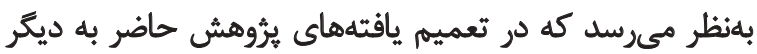

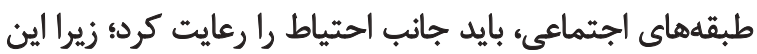

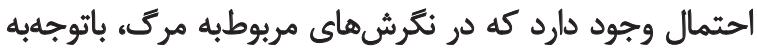

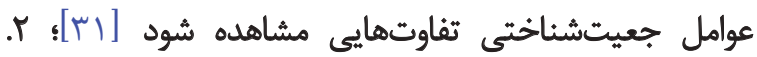

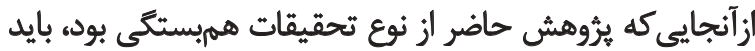

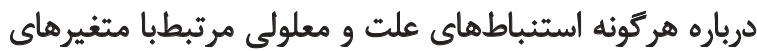
يُروهش احتياط كرد.
متغيرهاى مذهبي بررسى شده، متغيرهاي دلبستكّى نايمن به

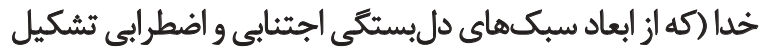

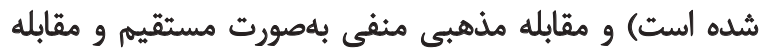

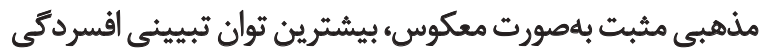

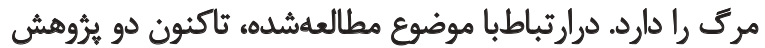

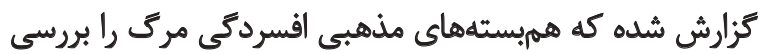

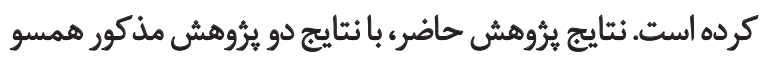

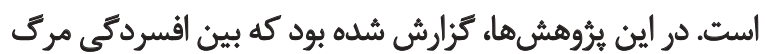

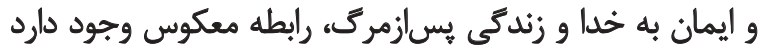

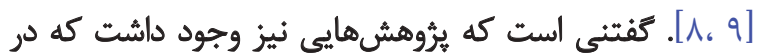

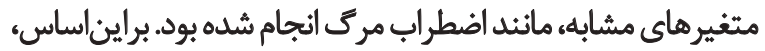

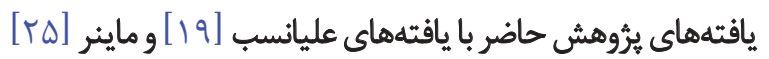

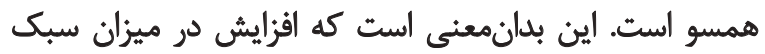

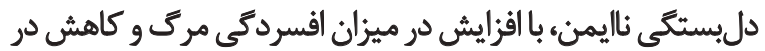

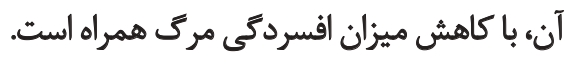

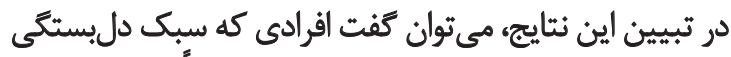

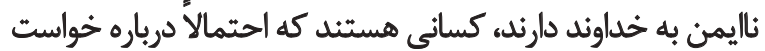

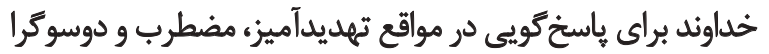

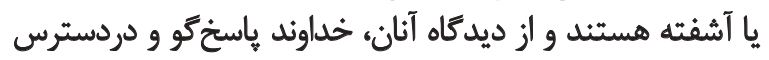

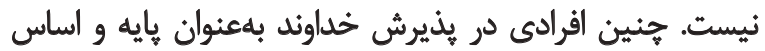

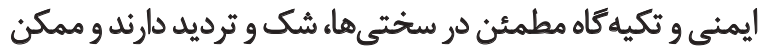

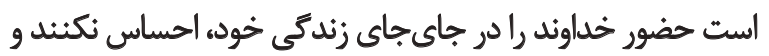

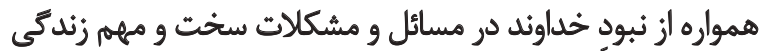

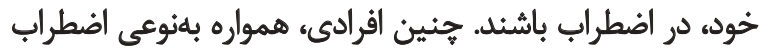

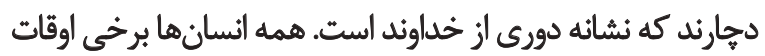

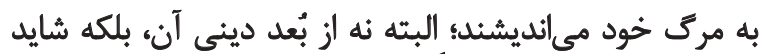

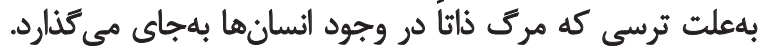

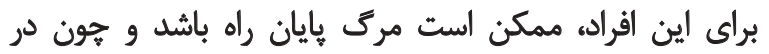

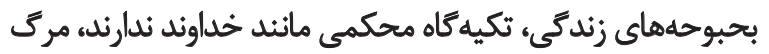

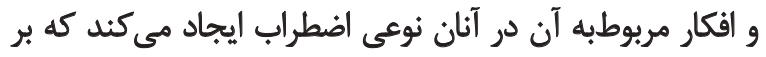

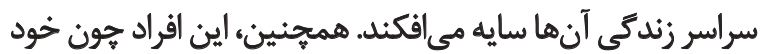

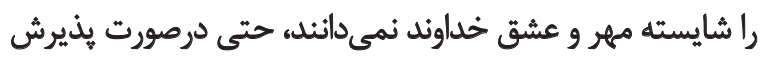

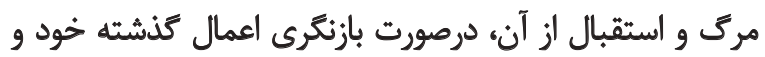

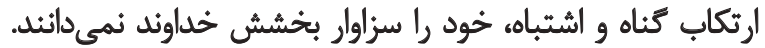

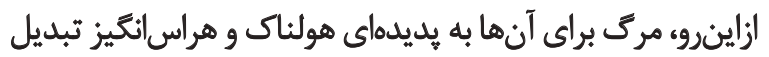

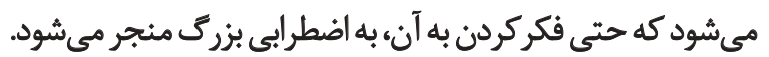
در سالهاى اخير در ميان محققان علوم اجتماعى و ورئان

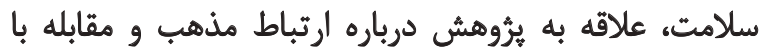

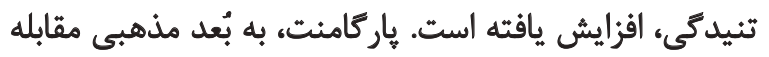

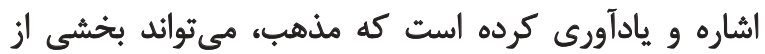

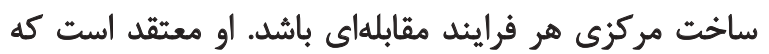

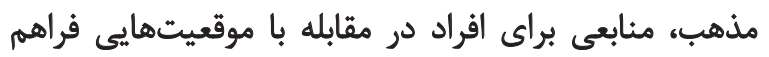

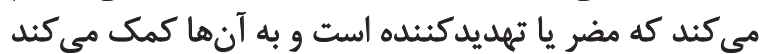




$$
\text { سباسكزן إزى }
$$

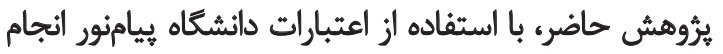

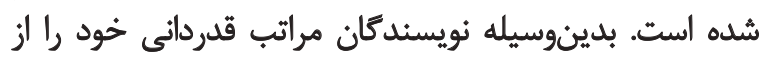

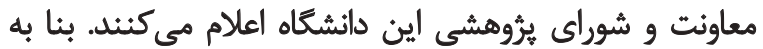

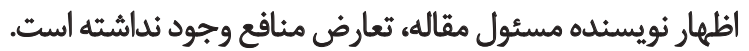




\section{References}

[1] Templer DI, Lavoie M, Chalgujian H, Thomas-Dobson S. The measure of death depression. Journal of Clinical Psychology. 1990; 46(6):834-839. doi: 10.1002/1097-4679(199011)46:6<834::aidjclp2270460623>3.0.co; $2-0$

[2] Cohen AB, Pierce JD, Chambers J, Meade R, Gorvine BJ, Koenig HG. Intrinsic and extrinsic religiosity, belief in the afterlife, death anxiety, and life satisfaction in young Catholics and Protestants. Journal of Research in Personality. 2005; 39(3):307-24. doi: 10.1016/j.jrp.2004.02.005

[3] Maxfield M, John S, Pyszczynski T. A terror management perspective on the role of death-related anxiety in psychological dysfunction. The Humanistic Psychologist. 2014; 42(1):35-53. doi: 10.1080/08873267.2012.732155

[4] Iverach L, Menzies RG, Menzies RE. Death anxiety and its role in psychopathology: Reviewing the status of a transdiagnostic construct. Clinical Psychology Review. 2014; 34(7):580-93. doi: 10.1016/j.cpr.2014.09.002

[5] Ongider N, Eyuboglu SO. Investigation of death anxiety among depressive patients. Journal of Clinical Psychiatry. 2013; 16(1):3446.

[6] Fortner BV, Robert A. Neimeyer B. Death anxiety in older adults: a quantitative review. Death Studies. 1999; 23(5):387-411. doi: $10.1080 / 074811899200920$

[7] Eshbaugh E, Henninger W. Potential mediators of the relationships between gender and death anxiety. Individual Differences Research. 2013; 11(1):22-30.

[8] Alvarado KA, Templer DI, Bresler C, Thomas-Dobson S. The relationship of religious variables to death depression and death anxiety. Journal of Clinical Psychology. 1995; 51(2):202-204. doi: 10.1002/1097-4679(199503)51:2<202::aid-jclp2270510209>3.0.co; 2-m

[9] Harville M, Stokes SJ, Templer DI, Rienzi B. Relation of existential and religious variables to the death depression scale-revised. OMEGA - Journal of Death and Dying. 2004; 48(2):165-84. doi: $10.2190 /$ c2mj-5dxn-eu6c-n2xr

[10] Pourghane P, Sharif Azar E, Zaer Sabet F, Khorsandi M. [Survey the effect of religious beliefs in stress reduction in students of Langroud Faculty of medical sciences (Persian)]. Journal of Holistic Nursing and Midwifery. 2011; 20(63):10-15.

[11] Pargament KI. The psychology of religion and coping: Theory, research and practice. New York: Guilford Press; 1997.

[12] Ghobari Bonab B. [Religious beliefs and its effects on mental health (Persian)]. Iranian Journal of Psychiatry \& Clinical Psychology. 1995; 1(4):48-54.

[13] Harding SR, Flannelly KJ, Weaver AJ, Costa KG. The influence of religion on death anxiety and death acceptance. Mental Health, Religion \& Culture. 2005; 8(4):253-61. doi: $10.1080 / 13674670412331304311$

[14] Soleimannejad A. [Fear of death in patients with hypochondriasis and anxiety disorders (Persian)]. Urmia Medical Journal. 2010; 21(2):273-279.

[15] Cassidy J, Shaver PR. Handbook of attachment: Theory, research and clinical application. $2^{\text {nd }}$ ed. NewYork: Guilford Press; 2008.
[16] Rowatt W, Kirkpatrick LA. Two dimensions of attachment to god and their relation to affect, religiosity, and personality constructs. Journal for the Scientific Study of Religion. 2002; 41(4):637-51. doi: 10.1111/1468-5906.00143

[17] Sepah Mansour M, Shahabi Zadeh F, Khoshnevis E. [Perceived childhood attachment, adult attachment and attachment to God (Persian)]. Journal of Developmental Psychology. 2008; 4(15):253265.

[18] Bradshaw M, Ellison CG, Marcum JP. Attachment to God, images of God, and psychological distress in a nationwide sample of Presbyterians. International Journal for the Psychology of Religion. 2010; 20(2):130-47. doi: 10.1080/10508611003608049

[19] Ulya-Nasab SH. [Investigating the relationship of piety and death anxiety among seminary and university students in Qom city (Persian)]. Ravanshenasi Va Din. 2010; 3(1):55-68.

[20] Aghazadeh SE, Mohammadzadeh A, Rezaie A. [Validation of death depression scale among Tabriz universities students (Persian)]. Journal of Research in Behavioural Sciences. 2014; 12(3):433-442.

[21] Golzari M. [Scale for measuring practice on religious Beliefs (Persian)]. Paper presented at: The Conference of Religion and Mental Health; 2000 December 14; Tehran, Iran.

[22] Pargament K, Feuille M, Burdzy D. The brief RCOPE: Current psychometric status of a short measure of religious coping. Religions. 2011; 2(4):51-76. doi: 10.3390/rel2010051

[23] Beck R, McDonald A. Attachment to God inventory. PsycTESTS Dataset. New York: American Psychological Association. 2004; doi: $10.1037 /$ t46035-000

[24] Taghiyareh F, Mazaheri MA, Azadfallah P. [The relationship between ego development, attachment to God and religious orientation in university students (Persian)]. Journal of Psychology. 2005; 9(1):34-50.

[25] Miner M. The impact of child- parent attachment, attachment to God and religious orientation on psychological adjustment. Journal of Psychology and Theology. 2009; 37(2):114-124.

[26] Pargament KI. Religion and coping: The current state of knowledge. In: Folkman S editor. Oxford Handbook of Stress, Health, and Coping. New York: Oxford University Press; 2011.

[27] Hasani Vajary K, Bahrami EH. [The role of religious coping in the explanation of spiritual happiness and mental health (Persian)]. Journal of Psychology. 2005; 9(3):248-260

[28] Pargament KI, Smith BW, Koenig HG, Perez L. Patterns of positive and negative religious coping with major life stressors. Journal for the Scientific Study of Religion. 1998; 37(4):710-724. doi: $10.2307 / 1388152$

[29] Bay PS, Beckman D, Trippi J, Gunderman R, Terry C. The effect of pastoral care services on anxiety, depression, hope, religious coping, and religious problem solving styles: A randomized controlled study. Journal of Religion and Health. 2007; 47(1):57-69. doi: 10.1007/s10943-007-9131-4

[30] Tarakeshwar N, Pargament KI. Religious coping in families of children with autism. Focus on Autism and Other Developmental Disabilities. 2001; 16(4):247-60. doi: 10.1177/108835760101600408

[31] Fessler DMT, Navarrete CD. The effect of age on death disgust: Challenges to terror management perspectives. Evolutionary Psychology. 2005; 3:279-296. doi: 10.1177/147470490500300120 